\author{
S. Sisó · C. Navarro · D. Hanzlíček · M. Vandevelde
}

\title{
Adult onset thalamocerebellar degeneration in dogs associated to neuronal storage of ceroid lipopigment
}

Received: 13 May 2004/ Revised: 17 June 2004 / Accepted: 18 June 2004/Published online: 8 September 2004

(C) Springer-Verlag 2004

\begin{abstract}
Late onset of hereditary cerebellar cortical abiotrophy has been described in a large variety of canine breeds. In some reported conditions, the cerebellar lesion is combined with degeneration of other systems. Here we describe a new hereditary cerebellar cortical degeneration in eight adult American Staffordshire and Pit Bull Terriers. The neuronal degeneration in these animals not only affects Purkinje cells of the cerebellum but also certain thalamic nuclei. In addition, nerve cell loss appears to be associated with a lysosomal storage disease, which is restricted to the affected cell populations. The stored material was histologically and ultrastructurally identified as fluorescent lipopigment. Since animals were euthanized at various stages of the disease, it could be shown that lysosomal storage preceded neuronal loss. Selective involvement of restricted neuronal populations is highly unusual in ceroid lipofuscinoses. It remains to be determined if the present neurodegenerative disease is caused by a primary or secondary neuronal ceroid lipofuscinosis.
\end{abstract}

\footnotetext{
S. Sisó $(\bowtie) \cdot M$. Vandevelde

NeuroCenter, Department of Clinical Veterinary Medicine, University of Bern,

Bremgartenstrasse 109A,

3012 Bern, Switzerland

E-mail: silvia.siso@itn.unibe.ch

Tel.: +41-31-6312206

Fax: + 41-31-6312538

C. Navarro

Hospital Meixoeiro,

Boucioa 36214 Vigo,

Pontevedra, Spain

D. Hanzlíček

Department of Clinical Veterinary Medicine,

University of Bern,

Bremgartenstrasse 109A, 3012 Bern,

Switzerland
}

Keywords Cerebellar cortical abiotrophy - Thalamic degeneration $\cdot$ Dogs $\cdot$ Ceroid lipofuscinoses

\section{Introduction}

Cerebellar degenerations are the largest group of neurodegenerative diseases in domestic animals. At least 40 entities in different species and breeds have been described, most of which are hereditary and of early onset [2]. However, a few cerebellar atrophies occur late in life. In some canine breeds, cerebellar degeneration in combination with degeneration of other neural systems such as the cerebral cortex, the striatum or autonomic nervous system occur [16]. The underlying cause is unknown in most of them, except for those in which degenerations has been associated to a lysosomal storage disease.

Recently, a late onset cerebellar cortical abiotrophy has been described in three American Staffordshire Terriers (AST) [6] and detected in three American Pit Bull Terriers (APBT) (de Martino et al., in preparation). Loss of Purkinje cells (Pc) as well as depletion of the granular cell layer was found. Animals were presented with gait abnormalities, generalized cerebellar ataxia with hypermetria, stiffness and loss of balance beginning between 2 and 5 years of age. We detected 40 AST and 8 APBT affected by such cerebellovestibular signs (unpublished data). The segregation analysis suggested a complex mode of inheritance with an important effect of a major gene in AST (Hanzlíček et al., in preparation).

We have had the opportunity to study eight cases in which we performed light microscopical, immunocytochemical and ultrastructural investigations. We found that the neuronal degeneration in these animals not only affects Pc of the cerebellum but also certain thalamic nuclei. In addition, nerve cell loss appears to be associated with a lysosomal storage disease, which is restricted to the affected cell populations. We identified the storage product as fluorescent lipopigment. 


\section{Materials and methods}

Light microscopy and immunohistochemical processing

Five AST and three APBT, of both sexes, were presented with slowly progressive cerebellovestibular signs beginning between 2 and 5 years and were euthanized between the age of 4-8 years and submitted to necropsy. The brains of each animal, and the spinal cord and peripheral nerves of three, were fixed in $10 \%$ buffered formalin saline solution ( $4 \%$ formaldehyde). In two dogs, samples from muscle, liver, pancreas, retina and small intestine were also processed for hematoxylin and eosin (HE) staining. Representative areas of the brain were embedded in paraffin, cut at $5 \mu \mathrm{m}$ and stained with HE, Luxol-fast blue (LFB), periodic acid-Schiff (PAS), Sudan black B and Bielschowsky. Glial fibrillary acidic protein (GFAP), synaptophysin (SYN), parvalbumin (PARV), ubiquitin (UBI), and 200-kDa phosphorylated and non-phosphorylated neurofilaments (NF-200) were immunohistochemically studied in each dog, except in case 8 . Matched representative brain sections of two normal dogs, aged 2.5 and 6 years, were included in the study as controls. For immunohistochemistry (IHC), $4-\mu \mathrm{m}$-thick sections were collected onto pre-treated glass slides (SuperFrost Plus, Menzel-Glaser) and baked overnight at $60^{\circ} \mathrm{C}$. The slides were then dewaxed and submitted to citrate buffer pretreatment designed to denature proteins (to unmask antigen epitopes) for PARV and NF-200 IHC. Endogenous peroxidase activity was inhibited with $0.03 \%$ hydrogen peroxide (Merck) for $15 \mathrm{~min}$ followed by washing in distilled water. Slides were incubated with $20 \%$ normal goat sera in PBS for 20 min prior to application of polyclonal antibodies, or with $20 \%$ normal swine sera for $20 \mathrm{~min}$ for monoclonal antibodies, and then incubated overnight at $4^{\circ} \mathrm{C}$ with anti-GFAP (Dako, 1:800), anti-UBI (Dako, 1:800), anti-NF-200 (Sigma, 1:800) and antiPARV (Sigma; 1:1500) polyclonal antibodies, and antiSYN (Dako, 1:200) monoclonal antibody. After washing, the sections were processed with the Chemate Detection Kit Peroxidase/DAB Rabbit/Mouse (Dako) following the instructions of the supplier. The slides were weakly counterstained with aqueous hematoxylin before mounting (Dako Glycergel).

\section{Electron microscopy}

Selected areas of frontal cerebral cortex, thalamic nuclei and lateral geniculate body of two AST (cases1 and 8) were cut into small cubes, fixed in $2.5 \%$ glutaraldehyde for 4-6 h, washed well in cacodylate buffer, post-fixed in $2 \%$ osmium tetroxide for $2 \mathrm{~h}$, dehydrated in graded alcohols and routinely embedded in Epon. Semithin sections were stained with toluidine blue and examined with light microscopy for selection of representative areas. Ultrathin sections were mounted on bared copper grids, stained with uranyl acetate and lead citrate, and examined with a Philips CM100 transmission electron microscope. An average of four blocks was examined for each case.

\section{Results}

Histopathological and immunohistochemical results

At necropsy, significant abnormalities were only found in the brain. Gross abnormalities included various degrees of atrophy of the cerebellum, inconsistent unilateral enlargement of lateral ventricles and inconsistent mild thalamic atrophy (Fig. 1A). Dogs were sorted into three stages according to the duration and grade of clinical signs: early stage, euthanized within 1 year after onset of clinical signs; advanced stage, after 1- to 3-year duration of clinical signs; terminal stage, after more than 3 years of clinical signs.

In the dogs that were euthanized at the early stage (cases 1 and 2), there was moderate loss of Pc. Most Pc and large numbers of thalamic neurons of the anteroventral and anteromedial nuclei, lateral anterior nuclei, lateral corpus geniculate nuclei and posterior nuclei were swollen and showed accumulation of sudanophilic material in cytoplasmic granules. The storage material displaced the nuclei and Nissl substance to the periphery of the cell. The material was PAS and Sudan black positive and showed yellow-green autofluorescence under UV light (Fig. 1C-E). Numerous macrophages containing autofluorescent material occurred in affected areas. Many neurons in various areas of the CNS displayed ageing pigment (lipofucin), which was only weakly autofluorescent compared to the affected neuronal populations and was not positive with PAS or Sudan black stains. IHC for SYN, a presynaptic protein, revealed that synapses to Pc were slightly decreased. NF200 IHC revealed dystrophic axons in the cerebellar nuclei $(\mathrm{Pc})$ and occasionally in the granule cell layer, decreased numbers of $\mathrm{Pc}$ dendrites in the molecular layer, but no cytoskeletal abnormalities in their soma. Correspondingly, PARV IHC of the Pc showed a intensity of expression similar to that of normal controls, but decreased dendritic staining (Fig. 2B). GFAP IHC revealed a moderate diffuse astrocytosis in the cerebellar cortex and the cerebellar deep nuclei $(\mathrm{CDN})$ (data not shown).

Dogs that were euthanized at the advanced stage of the disease (cases 3 and 4) showed widespread loss of Pc, depletion of granule cells (Fig. 1B) but preservation of basket cells. In affected thalamic nuclei, neuronal cell loss became apparent. Fluorescent lipopigment was present in both degenerating cell populations. Neurons of vestibular nuclei showed vacuolar degeneration while those of the cerebellar nuclei did not. GFAP staining revealed hypertrophic astrocytes with long processes surrounding Pc and granule cells. Synapses in the granular layer appeared decreased on SYN-immuno- 
Fig. 1 A Gross pathology of case 8 , showing marked cerebellar atrophy. B

Histological image of advanced case, showing severe loss of Purkinje cells and depletion of granule cells; HE staining. C, D Thalamic neurons of the anteroventral nucleus are swollen and show accumulation of PAS-positive (C) and sudanophilic (D) material in cytoplasmic granules. $\mathbf{E}$ Yellow-green autofluorescence under UV light in a Purkinje cell. Original magnification $\mathbf{B}$ $\times 100 ; \mathbf{C}-\mathbf{E} \times 1,000$
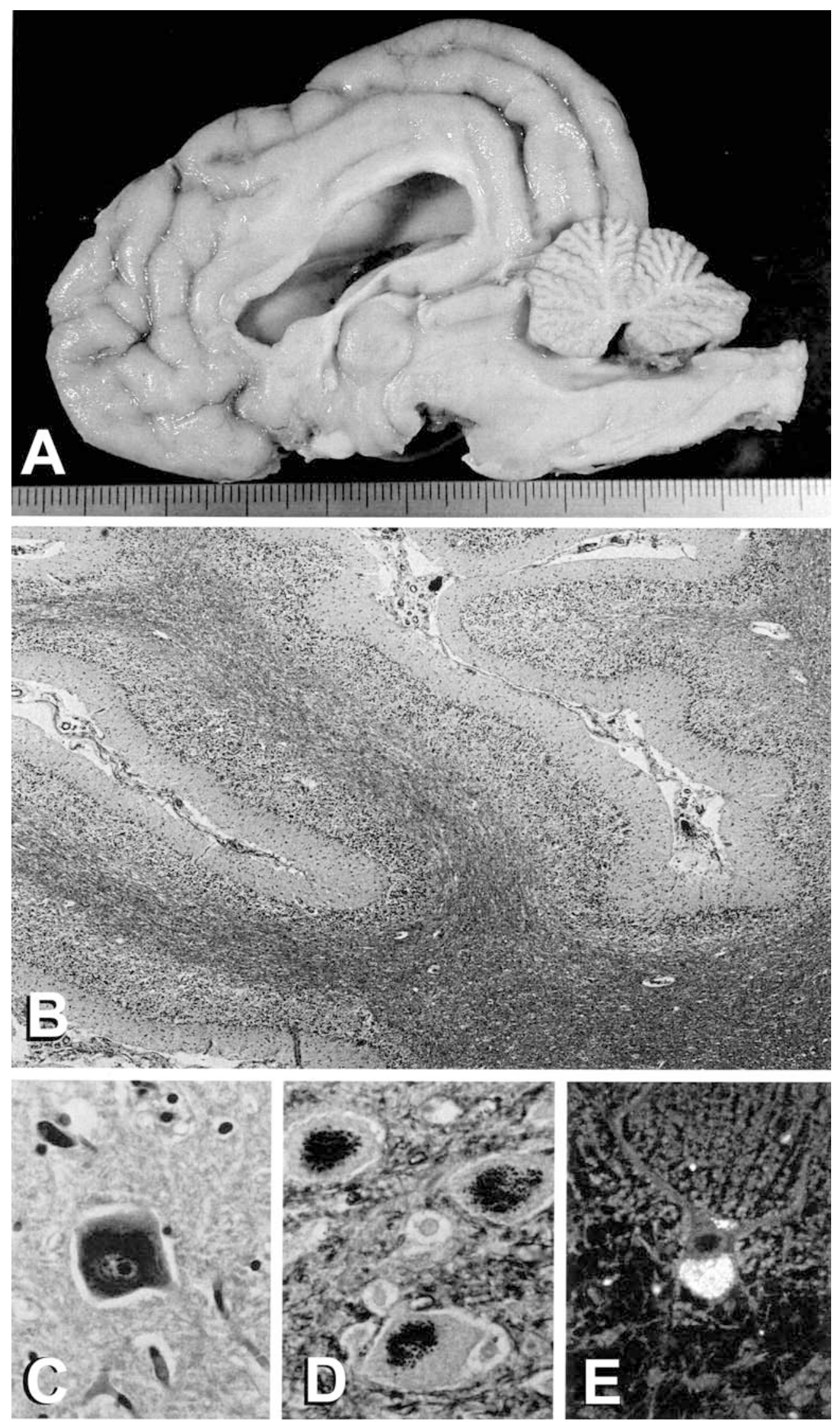

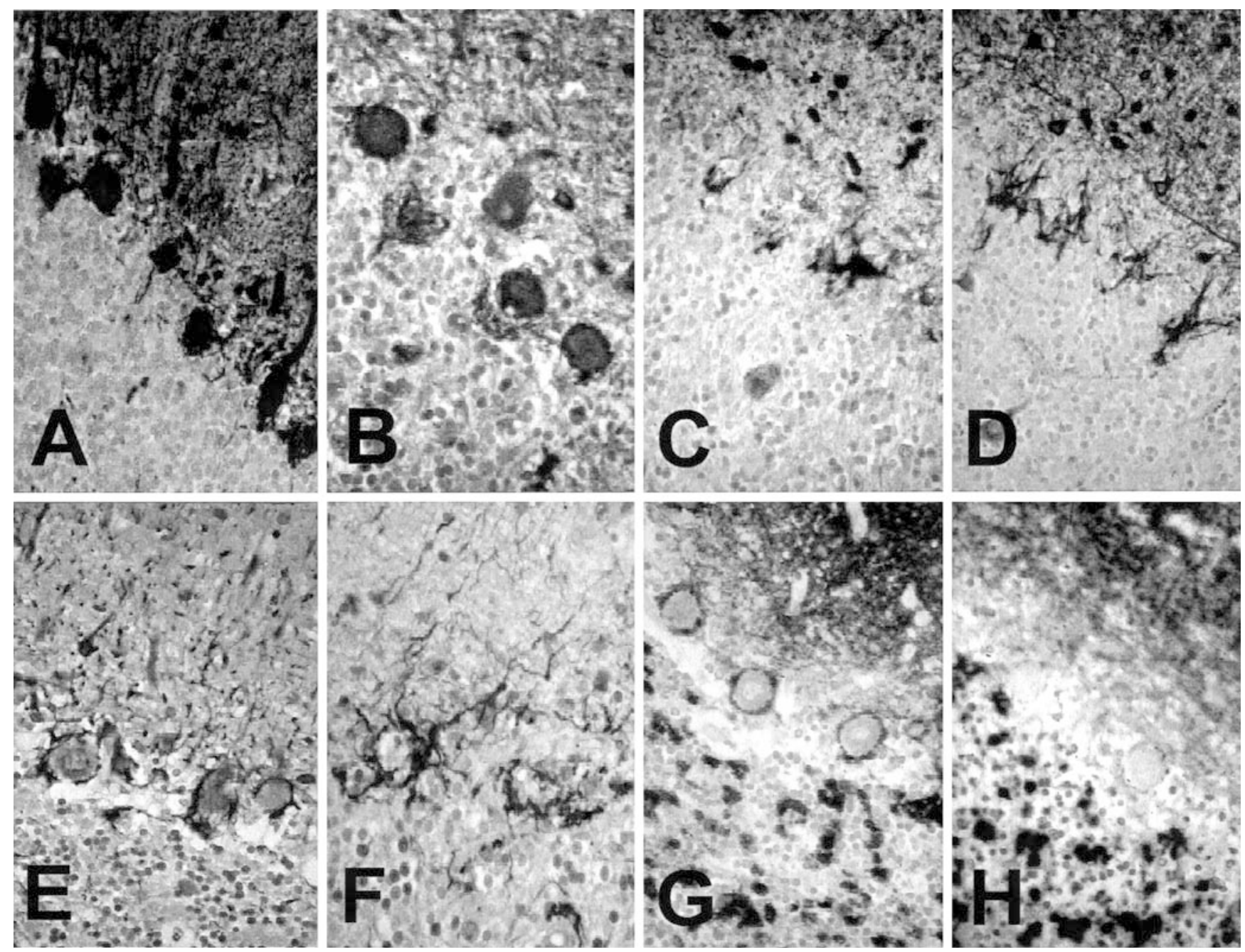

Fig. 2 Immunohistochemical results. A-D PARV staining: normal control (A), progressive loss of Purkinje cell processes (B), Purkinje cell somata $(\mathbf{C})$, preservation of basket cell staining in terminal stage (D). E, F NF-200 staining: normal control (E), preserved basket cell staining in terminal stage $(\mathbf{F})$. G, H SYN staining: normal control $(\mathbf{G})$, loss of staining around Purkinje cells in advanced stage (H) (PARV parvalbumin, $N F-200 \quad 200-\mathrm{kDa}$ phosphorylated and non-phosphorylated neurofilaments, $S Y N$ synaptophysin). Original magnification $\mathbf{A}-\mathbf{H} \times 400$

stained sections but remaining Pc were still labeled (Fig. 2H). NF-200 revealed that Pc still maintained their afferent collateral connections, while their dendrites and axons had almost disappeared. Basket cell processes surrounding Pc, which were observed by PARV IHC, were still preserved even when Pc had already disappeared (Fig. 2C). Some NF-200-positive torpedoes were found in the granular layer (Fig. 3B) as well as abundant strongly PARV-positive spheroids in the central and foliated white matter. There were no apparent cytoskeletal abnormalities in the soma of remaining Pc, Golgi cells or basket cells. GABAergic PARV-positive neuronal processes in the molecular layer were apparently preserved, while axons of the white matter were extremely reduced. GFAP IHC disclosed a severe astrocytosis in the CDN.

In dogs with the longest survival time (cases 5-8), nearly complete absence of Pc bodies, axons and dendrites was observed. There was severe depletion of granule cells, while basket cells were only multifocally decreased. The molecular layer was notably thinner. Occasionally, intracytoplasmic vacuolar degeneration of neurons in the CDN and inferior olivary nucleus due to transynaptic degeneration was observed. Remaining Pc, granule cells and CDN hardly showed labeling with SYN. The molecular layer showed reduced numbers of synapses, while large numbers of SYN-positive spheroids were found in the white matter of the cerebellum. NF-200 IHC revealed preserved basket cell arrangements (Fig. 2F). In addition, NF-200 and PARV IHC demonstrated decreased numbers of axons in the molecular and granular layers, and cerebellar white matter. PARV IHC demonstrated global loss of the inhibitory GABAergic neuronal population of the cerebellum, although a few immunolabeled basket cell arrangements were still present (Fig. 2D). Increased expression of UBI was found within macrophages and as granular and patchy intracytoplasmic inclusions in several remaining Pc (Fig. 3A). Affected thalamic nuclei exhibited diffuse nerve cell loss and gliosis. 
Fig. 3 A Ubiquitinated inclusions in the cytoplasm of remaining Purkinje cells in terminal stage of disease; UBI staining. B Torpedo in vicinity of a Purkinje cell at advance stages of disease; NF-200 staining (UBI ubiquitin). Original magnification $\mathbf{A}$ $\times 1,000 ; \mathrm{B} \times 400$
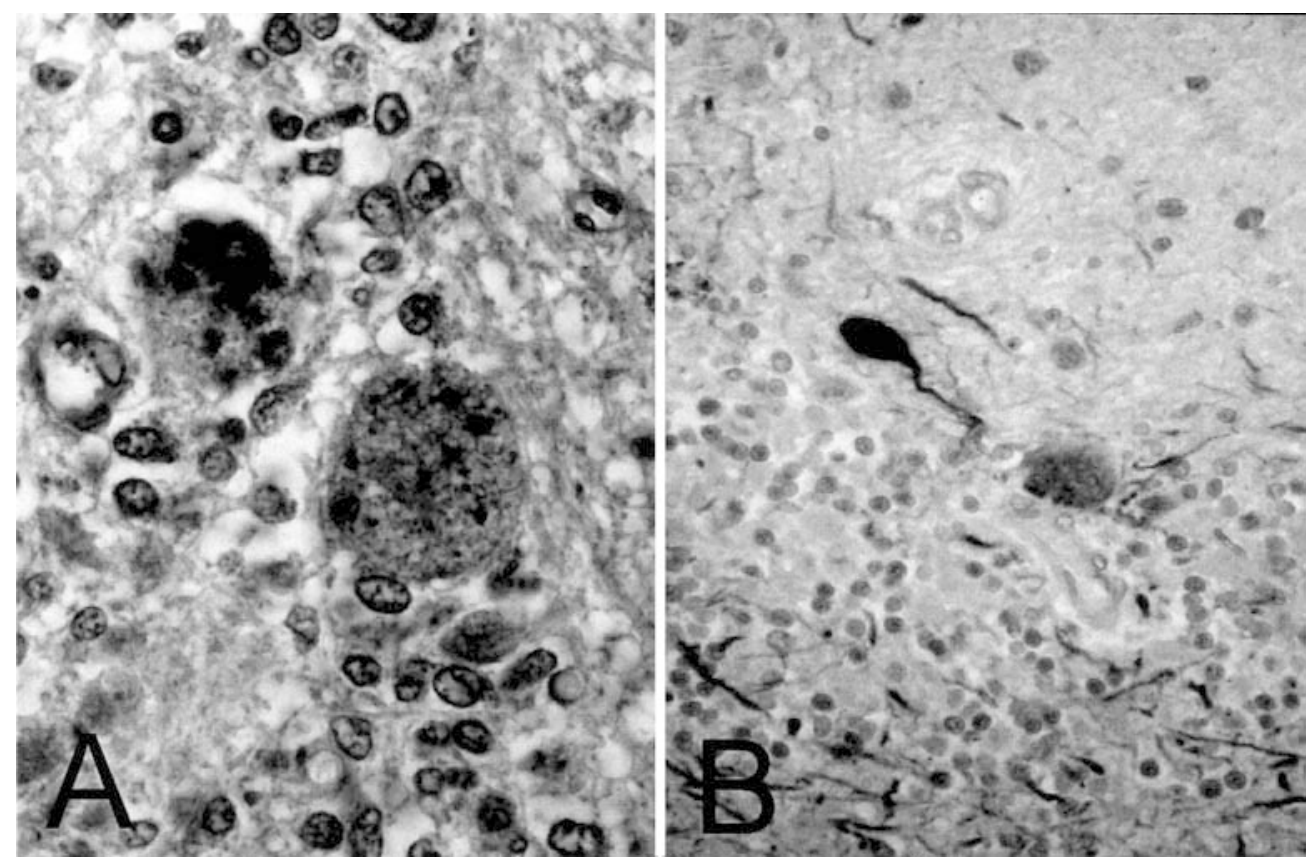

No other lesions were observed in the rest of the brain, in the spinal cord or in the peripheral nerves in any of the examined dogs.

\section{Ultrastructural findings}

Ultrastructural findings were similar in the two cases examined. In the thalamic nuclei and lateral geniculate body, several ballooned neurons were depicted in the preliminary examination of semithin sections. Ultrastructurally, neuronal cytoplasm was occupied by abnormal lysosomes, round or oval in shape, with diameters ranging from 0.8 to $2 \mu \mathrm{m}$ (Fig. 4A). These inclusions were membrane bound, of medium electron density, and formed of curved, straight or concentric profiles with alternating clear and dense bands of an approximate periodicity of 4-7 nm (Fig. 4B). Occasionally, normal lipofuscin pigment could be observed within the same neuron. The area occupied by abnormal lysosomes ranged from 150 to $200 \mu \mathrm{m}$. Golgi apparatus was prominent and ribosomes numerous, as usually seen in neurons. Mitochondria were of normal appearance and number. Macrophages filled with complex lipids, pseudo-myelin figures and other nonspecific inclusions were also observed, related or not to blood vessel walls. In general, neurons from cortical areas did not show significant changes.

\section{Discussion}

In the present study, we examined a series of eight dogs affected by an inherited neurodegenerative disorder with late onset Pc loss in the cerebellum and loss of neurons in certain thalamic nuclei. Hereditary Pc degeneration has been described in a large variety of domestic animals and breeds [2]. In some breeds, the cerebellar lesion was combined with degeneration of other systems, for example the striatum in Kerry Blue Terriers [12]. Recently, a cerebellar cortical degeneration has been described in AST [13]. The latter condition, which was thought to be similar to spinocerebellar ataxias in humans, shares many clinical (age, onset and course) and histopathological features (cerebellar cortical degeneration) with our cases. Nevertheless, Olby et al. [13] found neither thalamic involvement nor evidence of lysosomal storage within degenerating neurons. While simultaneous cerebellar and thalamic degeneration has been found in humans [15], to our knowledge, the combination of cerebellar with thalamic degeneration as seen in our material has not been reported before in animals.

The pathogenesis remains unknown in nearly all cerebellar degenerations in domestic animals. In the dogs of the present study, neuronal degeneration appeared to be associated with the presence of an adultonset neuronal ceroid lipofuscinoses (NCL). The diagnosis of NCL was based on detecting accumulation of yellow-brown granules within neurons, which exhibited yellow autofluorescence under ultraviolet light, and were PAS and Sudan black positive. These features are characteristic of NCL in humans [5] and animals [8]. The ultrastructural findings with lysosomal complex lamellar profiles within neurons are also consistent with a diagnosis of NCL. NCL represents a complex group of inherited neurodegenerative storage diseases in humans and several domestic animal species, characterized by accumulation of autofluorescent lipopigment material in neurons and other cells of the body followed by cell degeneration $[5,8,9]$. The stored material is ultrastructurally defined as lamellar lysosomal profiles, granular osmiophilic deposits, curvilinear bodies or fingerprint profiles, and apparently similar in composi- 

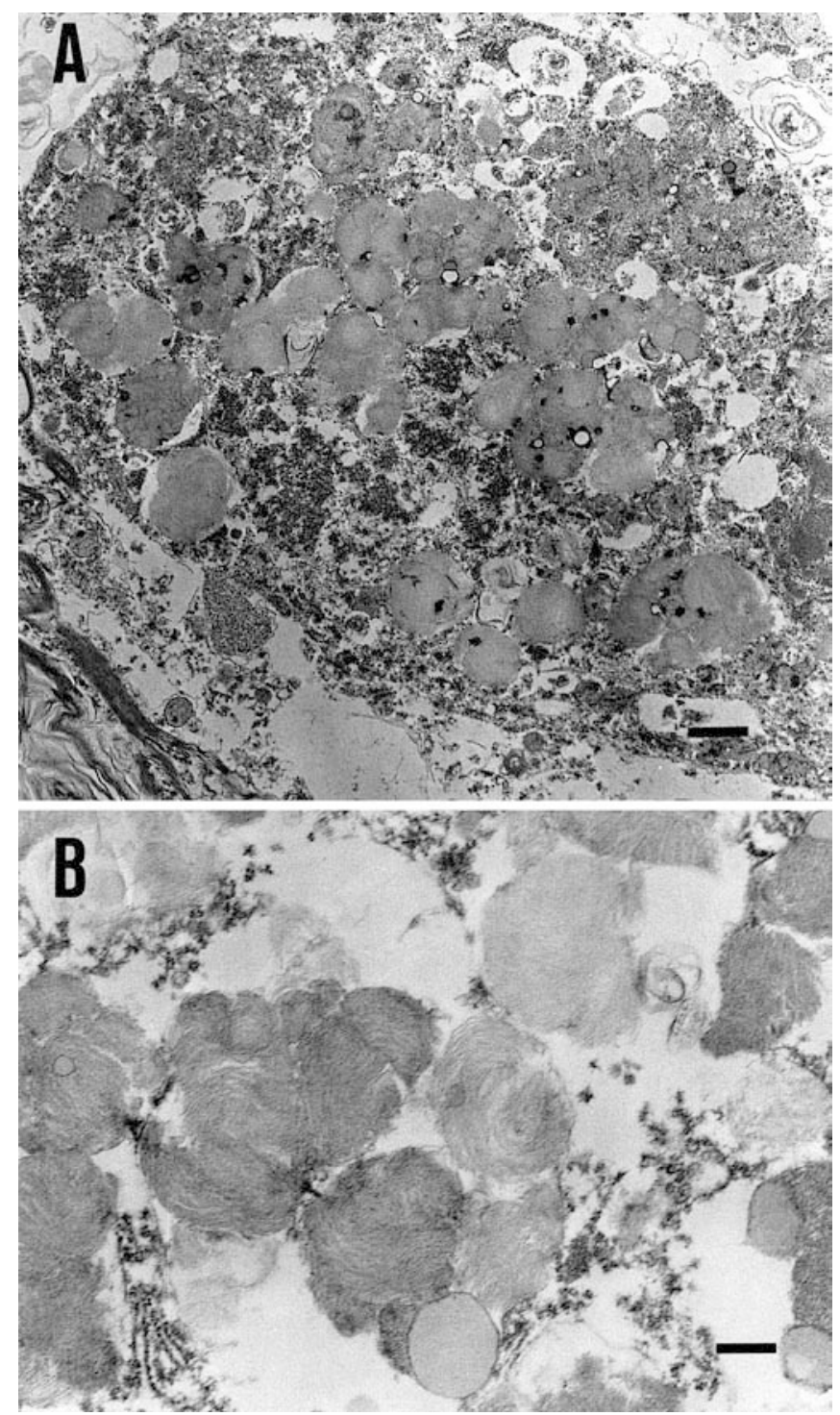

Fig. 4 Electron microscopy. A Ballooned neurons from the lateral geniculate body in case 1. Abnormal lysosomes of medium electron density fill the cytoplasm. Golgi apparatus, ribosomes and mitochondria are better seen at the periphery of the soma. B At higher magnification, concentric or curved profiles are seen within lysosomes, with alternating clear and dense bands of a periodicity of approximately 4-7 nm. Bars A $2.5 \mu \mathrm{m}$, B $1 \mu \mathrm{m}$

tion to the ageing pigments ceroid and lipofuscin. In humans, NCL have been classically classified into infantile, late infantile, juvenile (Batten disease) and adult (Kufs disease) types, according to the age of onset and the ultrastructural morphological features of the storage material $[5,11]$. Current classification of human NCL is based on eight main genetic forms (CLN1-8) [5]. Unfortunately, only few genetic and biochemical studies have so far been performed in canine NCL. Jolly et al. [9] classified canine NCL into prepubertal, early adult and adult onset forms. In dogs, NCL has been described in more than 15 breeds $[1,16]$. Affected animals are usually young adults (around 1-2 years) at onset of clinical signs, although in some NCL cases dogs may be much older at onset of signs [1]. Signs are extremely variable, but usually include some form of abnormal behavior like aggression or depression, and visual impairment [1]. Grossly, the cerebrum and/or cerebellum may be atrophic [14].

In contrast to previously reported NCL in which accumulation of stored material within neurons and sometimes other tissues is widespread, only Pc and certain thalamic neurons showed ceroid lipofuscin-like material in the present dogs. None of the examined extraneural tissues showed storage material or presence of ceroid lipofuscin-containing macrophages. Clinically, this particular form of NCL is associated with a progressive cerebellar ataxia that correlates with the severity of the cerebellar atrophy. No behavior abnormalities or visual impairment were detected. The ultrastructural findings with lysosomal complex lamellar profiles within neurons are also consistent with a diagnosis of canine NCL.

Neuronal degeneration, particularly of the cerebellum, is a frequent feature in NCL [5]. It is assumed that neuronal loss in human NCL is related to energy-linked excitotoxic mechanisms leading to continued influx of calcium into the neuron [10]. Since the animals in our study were euthanized at different stages of disease, we were able to show that accumulation of storage material in neurons preceded neuronal degeneration. Our IHC results on SYN and NF-200 protein expression suggest that distal regions of $\mathrm{Pc}$ axons were the first to degenerate. Later on, more proximal regions of these axons became involved with occurrence of large PARV-positive spheroids in the vicinity of Pc. We also found loss of Pc dendrites and presence of UBI inclusions in the cytoplasm. The exact mechanism of cell death remains unclear. While a causal relationship between thalamocerebellar degeneration and NCL seems plausible in our dogs, we have no explanation for the highly selective distribution of the defect. To our knowledge, such a restricted NCL pattern has not been described before in animals.

The question must be asked whether this disorder in the AST and APBT, is indeed a primary storage disease. In contrast to most other storage diseases, the underlying pathogenesis of NCL has not been entirely clarified. Sphingolipid activator protein (SAP, or saposins) storage has been found in the granular variant of human infantile-type NCL [7]. SAP material has been identified in adult canine NCL in miniature Schnauzers [8]. Some studies suggested that the primary defect in NCL might involve mitochondria rather than lysosomal catabolism $[7,10]$. According to recent investigations, accumulation of subunit c of mitochondrial ATP synthase (SCMAS) is commonly present in human NCL type, suggesting a delayed mitochondrial SCMAS degradation [3, 4, 5]. SCMAS accumulation has been described in a few cases of canine NCL [1]. There are morphological similarities between our cases and human adult NCL. Granular osmiophilic deposits, which are ultrastructurally characteristic of the SAP storage typically observed in the infantile type of human NCL (CLN1) [5], were not identified in the present cases. The lamellar profiles ob- 
served in our cases were suggestive of curvilinear forms, but classical fingerprint bodies observed in the adult type of human NCL (CLN4) [5] were not found in our cases. Studies for immunohistochemical detection of SCMAS, SAP and other mitochondrial enzymes are ongoing in these animals.

It is quite possible that the present disorder is not a primary NCL, but that the accumulation of fluorescent lipopigment was caused by a deficiency of the neuronal redox system in these particular cell populations. Further studies with more AST and APBT dogs at terminal stages of disease are necessary not only to confirm that neuronal degeneration is indeed restricted to a few cell populations regardless of progression of disease, but also that no other organs exhibit storage products. The similar biochemical properties of both affected cell populations, e.g., requirement of high energy supply, high numbers of mitochondria, etc., rather than, for example, neuroanatomical connections from the dentate nucleus to the ventrolateral and ventroanterior nuclei of thalamus [14], could explain this particular neurodegenerative combination.

Recent data in human spinocerebellar ataxias types 2 and 3 , which are fatal neurological disorders known as GAG repeat or polyglutamine diseases, suggest consistent thalamic involvement [15]. Of further interest is the fact that in a recent report [15] thalamic neuronal degeneration was associated with lipofuscin deposits. Progress in understanding the mechanism of the disease presented here may also require search for a triplet repeat disorder, which has not been found yet in domestic animals.

Acknowledgements We thank Valérie Juillerat and Christoph Pirsi for excellent technical assistance. We also would like to thank all private veterinarians for their willingness in providing the appropriate material.

\section{References}

1. Braund KG (2003) Storage disorders. In: Braund KG (ed) Clinical neurology in small animals-Localization, diagnosis and treatment. International Veterinary Information Service (www.ivis.org), Ithaca
2. De Lahunta A (1980) Comparative cerebellar disease in domestic animals. Compend Contin Educ 2:8-19

3. Elleder M, Sokolová J, Hrebícek (1997) Follow-up study of subunit $\mathrm{c}$ of mitochondrial ATP synthase (SCMAS) in Batten disease and in unrelated lysosomal disorders. Acta Neuropathol 93:379-390

4. Ezaki J, Wolfe LS, Higuti T, Ishidoh K, Kominami E (1995) Specific delay of degradation of mitochondrial ATP synthase subunit $\mathrm{c}$ in late infantile neuronal ceroid lipofuscinoses (Batten disease). J Neurochem 64:733-741

5. Haltia M (2003) The neuronal ceroid-lipofuscinoses. J Neuropathol Exp Neurol 62:1-13

6. Hanzlíček D, Kathmann I, Bley T, Srenk P, Botteron C, Gaillard C, Jaggy A (2003) Cerebellar cortical abiotrophy in American Staffordshire terriers: clinical and pathological description of 3 cases. Schweiz Arch Tierheilkd 145:369-375

7. Jolly RD (1995) Comparative biology of the neuronal ceroidlipofuscinoses (NCL): an overview. Am J Med Genet 57:307311

8. Jolly RD, Walkley SU (1997) Lysosomal storage diseases of animals: an essay in comparative pathology. Vet Pathol 34:527-548

9. Jolly RD, Palmer DN, Studdert VP, Sutton RH, Kelly WR, Koppang N, Dahme G, Hartley WJ, Patterson JS, Riis RC (1994) Canine ceroid-lipofuscinoses: a review and classification. J Small Anim Pract 35:299-306

10. Jolly RD, Brown S, Das AM, Walkley SU (2002) Mitochondrial dysfunction in the neuronal ceroid-lipofuscinoses (Batten disease). Neurochem Int 40:565-571

11. Lake BD (1997) Lysosomal and peroxisomal disorders. In: Graham DI, Lantos PL (eds) Greenfield's Neuropathology, 6th edn. Arnold, London, pp 657-753

12. Montgomery DL, Storts RW (1984) Hereditary striatonigral and cerebello-olivary degeneration of the Kerry Blue Terrier. II. Ultrastructural lesions in the caudate nucleus and cerebellar cortex. J Neuropathol Exp Neurol 43:263-275

13. Olby N, Blot S, Thibaud JL,Phillips J, O'Brien DP, Burr J, Berg J, Brown T, Breen M (2004) Cerebellar cortical degeneration in adult American Staffordshire Terriers. J Vet Intern Med 18:201-208

14. Palay SL, Chan-Palay V (1974) Cerebellar cortex. Cytology and organization. Springer, Berlin Heidelberg New York, pp 15

15. Rüb U, Del Turco D, Del Tredici K, Vos RAI de, Brunt ER, Reifenberger G, Seifried C, Schultz C, Auburger G, Braak H (2003) Thalamic involvement in a spinocerebellar ataxia type 2 (SCA2) and a spinocerebellar ataxia type 3 (SCA3) patient, and its clinical relevance. Brain 126:22572272

16. Summers BA, Cummings JF, Lahunta A de (1995) Veterinary neuropathology. Mosby, St Louis 